\title{
Nasal Polyposis and Its Association with Cardiac Functions
}

\author{
Sapna Dhiman ${ }^{\mathrm{a}}$ Abhishek Dhiman ${ }^{\mathrm{b}}$ Ramesh K. Azad ${ }^{\mathrm{a}}$ Prakash C. Negi ${ }^{\mathrm{c}}$ \\ Bhushan Lal ${ }^{\mathrm{a}}$ Jagdeep S. Thakur ${ }^{\mathrm{a}}$ \\ aDepartment of Otolaryngology-Head \& Neck Surgery (ENT), Indira Gandhi Medical College, Shimla, India; \\ bepartment of Radiodiagnosis, Indira Gandhi Medical College, Shimla, India; 'Department of Cardiology, Indira \\ Gandhi Medical College, Shimla, India
}

\section{Highlights of the Study}

- Upper respiratory airway obstruction is found to be associated with snoring and hypoxia, causing cardiac dysfunctions.

- We evaluated the effect of nasal polyps and of their treatment on cardiac functions.

- Significant improvement was observed in cardiac functions of subjects treated by sinus surgery.

\section{Keywords}

Nasal polyps · Sinusitis · Right ventricular function · Surgery

\section{Abstract}

Objective: The aim of the study was to ascertain the effect of nasal polyposis on cardiac functions. Material and Methods: A prospective randomized interventional open-label endpoint-controlled study was conducted in an academic tertiary care hospital. Thirty-one patients with chronic rhinosinusitis with nasal polyposis were enrolled and administered fluticasone furoate nasal spray for 3 weeks before randomly segregation into surgical or medical group. The treatment continued for 3 months in both groups. The SNOT-22 (SinoNasal Outcome Test-22) score, polyp grade, and right ventricular and pulmonary arterial functions were recorded in both groups before and after 3 months of the intervention. Results: Both groups had significant improvement in SNOT22 scores after 3 months of intervention. Both groups showed karger@karger.com www.karger.com/mpp

Karger $\stackrel{\text { ' }}{5}$
C 2021 The Author(s).

Published by S. Karger AG, Basel

This is an Open Access article licensed under the Creative Commons (http://www.karger.com/Services/OpenAccessLicense), applicable to the online version of the article only. Usage and distribution for commercial purposes requires written permission. Attribution-NonCommercial-4.0 International License (CC BY-NC) improvement in cardiac functions, but statistical significance was found only in subjects who underwent surgery. Conclusion: Nasal polyp affects cardiac functions, and this needs further evaluation and research through studies on large samples.

(c) 2021 The Author(s). Published by S. Karger AG, Basel

\section{Introduction}

Nasal polyposis causes obstructive nasal breathing, which is further augmented by a hyper-reactive mucosa in patients with chronic rhinosinusitis. Nasal obstruction with sleep-induced relaxation of nasopharyngeal muscles causes snoring and sleep apnoea in severe cases. This airway obstruction leads to hypoxia and hypercapnia that can lead to pulmonary arterial vasoconstriction and pulmonary hypertension. A previous study showed signifi-

Correspondence to:

Jagdeep S. Thakur, anujagdeep@yahoo.co.in 
cant effects of chronic adenotonsillar hypertrophy on cardiopulmonary functions that improved significantly after surgery [1]. Upper airway obstruction due to nasal polyposis has also been reported to be associated with pulmonary hypertension [2].

Patients with nasal polyposis are treated pharmacologically with steroids and/or endoscopic sinus surgery to relieve the obstruction. Both intranasal steroids and endoscopic sinus surgery have been found efficacious in mitigating symptoms, while the use of postoperative topical steroids has been found beneficial within the first year of surgery [3-5]. It has been observed in clinical practice that patients become reluctant to undergo endoscopic sinus surgery while using preoperative steroids due to improvement in symptoms or lack of expert counselling. This reluctance can put such patients at risk for cardiac dysfunctions, which could further worsen in the case of coexisting morbidities like asthma or hypertension. To prove this hypothesis, we conducted a prospective interventional study to assess the effect of nasal polyps and their treatment (either surgery or medical) on cardiac functions.

\section{Subjects and Methods}

\section{Study Design}

This prospective randomized, interventional, open-label, endpoint-controlled pilot study was conducted in the Department of Otorhinolaryngology, Head and Neck Surgery (ENT), and Cardiology after approval by the Institutional Ethics Review Board. Informed consent for this study was obtained from all subjects for this study.

\section{Sample Data}

Thirty-one adult subjects suffering from chronic rhinosinusitis with bilateral nasal polyps and matched for age (18-60 years), body mass index, and duration of disease were enrolled. We excluded patients with clinical features suggestive of altered cardiac functions or with various comorbidities known to effect cardiac functions such as sleep apnoea or history of snoring, hypertension, chronic kidney disease, diabetes, chronic obstructive airway disease, asthma, pulmonary thromboembolism, structural heart disease, left ventricular dysfunction were excluded. Subjects were randomized into surgical or medical group based on a computer-generated randomization sequence. All participants were subjected to detailed otorhinolaryngology and cardiology evaluation at the time of enrolment and after 3 months of the intervention.

Participants in both groups were administered fluticasone furoate nasal spray, 2 puffs $(27.5 \mathrm{mcg} /$ spray $)$ a day for 3 months while the surgical group were subjected to endoscopic sinus surgery after 3 weeks of medication that continued for 3 months in total. The surgical group consisted of 17 subjects with mean age of $35 \pm 11.8$ years while the medical group had 14 subjects with mean age of
$35.71 \pm 11.7$ years. ENT evaluation was done using Sino-Nasal Outcome Test-22 (SNOT-22) questionnaire tool, nasal endoscopy and computerized tomography. Polyps were graded during nasal endoscopic examination and high-resolution computerized tomography, using Lund Mackay scoring [6]. Right ventricular hypertrophy was diagnosed as per the standard ECG protocol and presence of any 2 criteria: QRS axis of $>900, \mathrm{R} / \mathrm{S}$ in V1 and V2 $>1$, $\mathrm{ST}$ and $\mathrm{T}$ wave depression in lead V1 and V2, S wave $>7 \mathrm{~mm}$ in V5 and V6.

\section{Right Ventricle Functions}

Two-dimensional echocardiography was performed in all subjects with an iE33 cardiovascular ultrasound machine (Phillips Medical Division, Andover, MA, USA) 2-5 MHz phased array probe in left lateral decubitus position. 2-D imaging in a modified four-chamber view was recorded with ECG gating to record right ventricle (RV) images in end diastole (RVED) and end systole (RVES) to estimate the RV area using planimetry methods to measure the percentage fractional shortening of RV area at end systole. $\mathrm{M}$ mode tracing was used at the lateral tricuspid annulus to record tricuspid annular peak systolic excursion (TAPSE) in the longitudinal axis. RV global longitudinal systolic strain (RVLSS) measured using speckle tracking method of recording 2-D images in a modified four-chamber view at the minimum of $50 \mathrm{~Hz}$ image acquisition frequency. The presence of tricuspid regurgitation (TR) was screened using colour flow mapping to record TR jet. TR velocity was measured using a continuous wave Doppler aligning TR jet with an incident sound wave beam for accurate measurement of the TR velocity. The pulse wave Doppler signals were recorded in right ventricular outflow tract to measure velocity time integral and pulmonary flow acceleration time (PFAT) from 3 consecutive Doppler envelops recorded gated with surface ECG. Right ventricular ejection fraction was calculated as right ventricular end diastolic volume minus right ventricular end systolic volume/right ventricular end diastolic volume/RVLSS. Mean pulmonary arterial pressure (MPAP) was measured from pulmonary flow acceleration time (PFAT) recorded across the right ventricular outflow track using the Dabestani-Mahan's formula (MPAP = 90 minus $0.62 \times$ PFAT). MPAP of more than $25 \mathrm{~mm} \mathrm{Hg}$ was used for labelling pulmonary arterial hypertension.

The data were recorded as mean \pm SD and the significance of differences in the distribution of categorical outcomes was compared using the $\chi^{2}$ test and continuous variables with normal distribution with the paired $t$ test between the 2 study groups.

\section{Results}

The study populations in both groups had no significant difference during enrolment, except body mass index, which was significantly higher in the medical treatment group $(21.71 \pm 3.63$ and $26.28 \pm 3.89$, surgical and medical group respectively, $p=0.002$ ). Five subjects in the medical group dropped out for the final follow-up and did not respond, despite repetitive telephonic requests. 
Table 1. Comparison of pre- and posttreatment parameters of medical and surgical arms

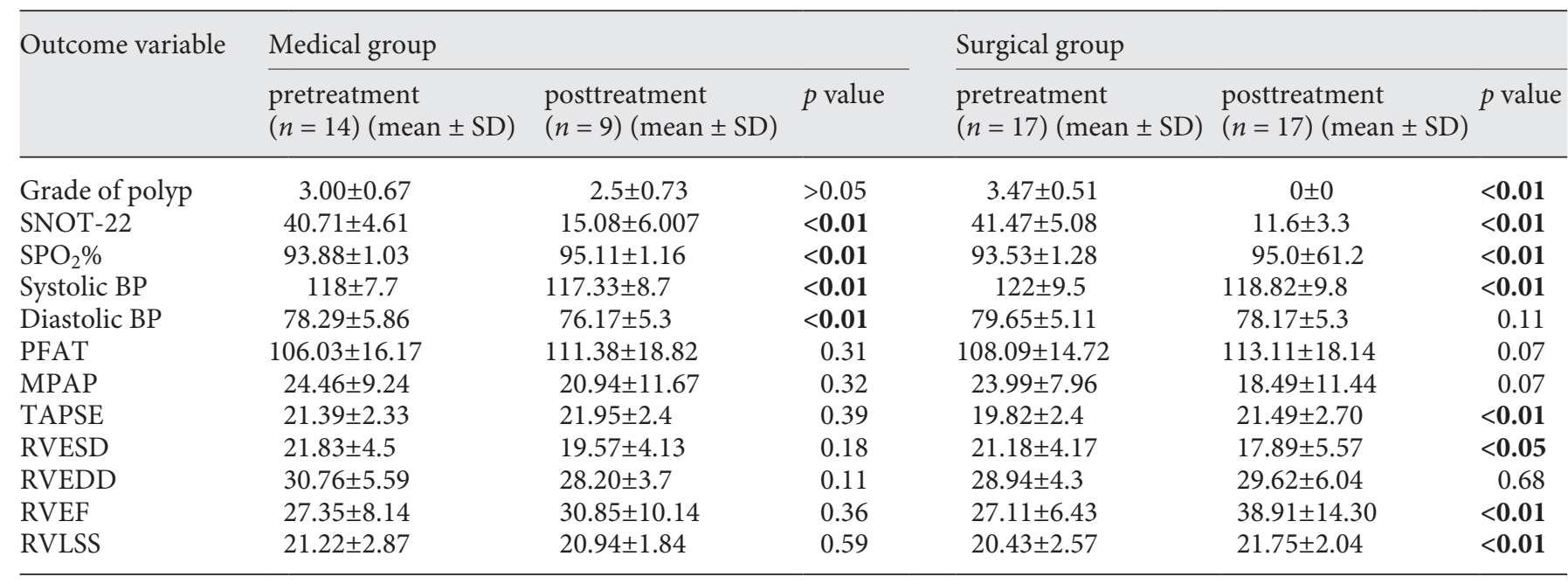

PFAT, pulmonary flow acceleration time (in milliseconds); MPAP, mean arterial pressure (in mm Hg); TAPSE, tricuspid annular plane systolic excursion (in millimetres); RVESD, right ventricular end systolic diameter (in millimetres); RVEDD, right ventricular end diastolic diameter (in millimetres); RVEF, right ventricular ejection fraction (in percent); RVLSS, right ventricular longitudinal systolic strain (in percent); SNOT-22, Sino-Nasal Outcome Test-22.

\section{Surgical Group}

SNOT-22, oxygen saturation score and systolic blood pressure parameters were improved significantly $(p<$ 0.05). RV function parameters like RVESD, TAPSE, and RVLSS also improved significantly $(p<0.05)$. The mean PFAT increased and PAP decreased from the baseline with $p<0.07$ (Table 1). Six patients had pulmonary hypertension at the time of enrolment, which had become normal in 3 subjects at the time of final follow-up.

\section{Medical Group}

We lost 5 subjects in this group at the time of final follow-up due to COVID-19 lockdown, and only 9 subjects were available for the final evaluation. These 9 subjects showed significant improvement $(p<0.05)$ in the SNOT22 score, oxygen saturation, and blood pressure after treatment. Although various RV parameters like TAPSE, RVEDD, and RVLSS improved after treatment, none had statistical significance. Similarly, pulmonary arterial functions (mean PFAT and PAP) also improved without any statistical significance after treatment (Table 1). This group also contained 6 patients with pulmonary hypertension as per the definition and of which 2 subjects had normal pulmonary arterial pressure after the treatment.

\section{Intergroup Analysis}

None of the baseline parameters showed any significant difference between groups, except body mass in- dex and polyp grade, which were higher in medical and surgical groups, respectively. The SNOT-22 score and nasal polyposis grade on CT scan were found to be significantly reduced in the surgical group as compared to the medical group $(p<0.05)$. Arterial oxygen saturation, mean pulmonary arterial pressure, and mean PFAT improved in both groups, but these were not found statistically significant in comparison with each other. RV systolic parameters RVEDD, RVESS, TAPSE, and RVLSS were also not found to be statistically significant between the 2 groups. However, these results need to be interpreted with caution due to loss of subjects in 1 group and higher body mass index in the medical group that caused an unequal distribution in the groups.

\section{Discussion}

Nasal polyposis is one of the most common causes of upper airway obstruction in adults and has an incidence of about $1-4 \%$ [7]. The present study was conducted to support the hypothesis that nasal polyps can lead to cardiac dysfunctions as chronic hypoxia leads to pulmonary arteriolar vasoconstriction that increases right ventricular systolic dysfunction, consequently right ventricular systolic dysfunction; furthermore, sinus surgery in comparison to conservative treatment tends 
to improve upper airway obstruction and improves cardiac functions.

We found significant improvement in a majority of cardiac parameters in the surgical group in comparison with the medical group after 3 months of intervention. Similar observations have been made in previous studies. Fidan et al. [2] conducted a study on 45 cases of nasal polyposis and 31 healthy subjects to determine the effect of endoscopic sinus surgery on mean pulmonary arterial pressure in extensive nasal polyposis. They found pulmonary hypertension in 26 patients with nasal polyposis at the time of enrolment and mean pulmonary arterial pressure had reduced significantly after 3 months of endoscopic sinus surgery. Lima et al. [7] evaluated pulmonary hypertension in 54 children suffering from adenotonsillar hypertrophy and 24 children with allergic rhinitis. They found significantly increased pulmonary arterial pressure in both groups. Similarly, Simsek et al. [8] conducted a cross-sectional observational study of 40 subjects with nasal polyp without any evidence of right ventricular dysfunctions. They found significantly altered right ventricular functions and its correlation with pulmonary arterial pressure and right ventricular parameters. Bayrak et al. [9] conducted a study on 35 subjects to assess the effect of allergic rhinitis on pulmonary functions. They found significant differences in cardiac functions during symptomatic to asymptomatic period. There was a positive correlation between mean pulmonary arterial pressure and nasal obstruction score. Taking this into account, chronic nasal obstruction with nasal polyposis could have much more effect on these parameters, requiring surgical intervention, especially in cases with other comorbidities. This is further proved by our study as numerous cardiac parameters were affected and which improved subsequently in surgical group that had a higher polyp grade in comparison to the medical group.

Our study did not find any significant improvement in cardiac functions parameters in the medical group. Contrary to our observations, Yuksel et al. [10] found significant reduction in pulmonary arterial pressure in 23 allergic rhinitis children treated with topical nasal steroid as compared to 19 healthy children. Similarly, Reisle et al. [11] also found significant improvement in mean pulmonary arterial pressure functions in 49 children with allergic rhinitis who had topical mometasone furoate for 8 weeks. However, these studies did not include subjects with nasal polyposis, which improved the pulmonary arterial pressure in the subjects even with topical medication. This further supports our hypothesis. A cross-sectional study showed a correlation between pulmonary arterial pressure and nasal patency in 183 mouthbreathing children suffering with allergic rhinosinusitis, and adenotonsillar hypertrophy. They found significant correlation between reduced nasal patency and raised pulmonary arterial pressure [12]. Similarly, Lima et al. [13] found significantly raised pulmonary arterial pressure in children with adenotonsillar hypertrophy and allergic rhinitis.

Topical nasal steroid and endoscopic sinus surgery are the standard treatment in nasal polyposis. In clinical practice, topical steroids are administered before surgery to reduce mucosal oedema that further gives symptomatic relief to the patients with SNOT-22, as validated by our study. Aukema et al. [14] conducted a doubleblind, placebo-controlled study in 28 patients with chronic rhinosinusitis with or without severe nasal polyp to assess the effect of fluticasone propionate on the probability of need for sinus surgery. They found mitigation of various symptoms and peak inspiratory flow after medication and hence advocated topical nasal spray in all cases planned for sinus surgery as it can decrease the need for surgery. Similarly, Stjärne et al. [15] found symptomatic mitigation in subjects with nasal polyps using mometasone furoate in a randomized double-blind placebo-controlled parallel group multicentric study. Our study also found significant improvements in SNOT-22 scores in both groups even though the surgical group had significantly better scores.

In our knowledge, this is the first study evaluating the effects of nasal polyps and its 2 standard treatment options on cardiac functions. We found significant improvement in SNOT-22 scores in both treatment groups, while cardiac functions improved in surgical group only. This can be explained on the basis of significantly better improvement in polyp scores and SNOT-22 scores in the surgical group than the medical group. Endoscopic sinus surgery improves nasal airway, thus decreasing hypoxia, which subsequently improves pulmonary artery flow as mentioned earlier. However, this did not result in any significant difference in cardiac functions in the 2 treatment groups. This might have occurred due to the unequal distribution of subjects as 5 subjects from the medical group were lost in the final follow-up. Moreover, higher body mass index in this group could also have influenced the analysis. A large sample data with a longer follow-up period may result in significant differences in all these parameters. 
The relevance of these cardiac parameters to an ENT surgeon is questionable, and evaluation in terms of minimal clinically important difference may have proved helpful for clinical research and practice. We partly agree with this argument. However, our pilot study highlights cardiac dysfunctions in nasal polyposis, which may not be apparent symptomatically, and we should be aware of them. The present study has the limitations of small sample size, body mass index bias, duration of study, and minimal clinical improvement difference in the methodology.

\section{Conclusion}

This pilot study found significant effects of nasal polyposis on cardiac functions that warrant further research using a rigorous methodology.

\section{Acknowledgement}

The authors acknowledge the contribution of the staff of the echocardiography laboratory in this research.

\section{Statement of Ethics}

This research complied with the guidelines for human studies and was conducted ethically in accordance with the World Medical Association Declaration of Helsinki.

\section{Conflict of Interest Statement}

The authors have no conflicts of interest to declare.

\section{Funding Sources}

The authors did not receive any funding.

\section{Author Contributions}

S. Dhiman and A. Dhiman were responsible for conception, data collection and analysis, and drafting of this manuscript; J.S. Thakur was responsible for conception, design, methodology, data analysis, and drafting of the manuscript; P.C. Negi, Ramesh Azad and Bhushan Lal were involved in conception, methodology, and critical revision of the manuscript. All authors have read and approved the final version.

\section{References}

1 Mahajan M, Thakur JS, Azad RK, Mohindroo NK, Negi PC. Cardiopulmonary functions and adenotonsillectomy: surgical indications need revision. J Laryngol Otol. 2016;130(12): $1120-4$.

2 Fidan V, Aksakal E. Effects of endoscopic sinus surgery on pulmonary artery pressure in patients with extensive nasal polyposis. J Craniofac Surg. 2011;22(2):592-3.

3 Rimmer J, Fokkens W, Chong LY, Hopkins C. Surgical versus medical interventions for chronic rhinosinusitis with nasal polyps. Cochrane Database Syst Rev. 2014;12(12): CD006991.

4 Kalish L, Snidvongs K, Sivasubramaniam R, Cope D, Harvey RJ. Topical steroids for nasal polyps. Cochrane Database Syst Rev. 2012;12: CD006549.

5 Fandiño M, Macdonald KI, Lee J, Witterick IJ. The use of postoperative topical corticosteroids in chronic rhinosinusitis with nasal polyps: a systematic review and meta-analysis. Am J Rhinol Allergy. 2013 SepOct;27(5): e146-57.
6 Lund VJ, Mackay IS. Staging in rhinosinusitus. Rhinology. 1993;31(4):183-4.

7 Lima MS, Nader CMFF, Franco LP, Meira ZMA, Capanema FD, Guimarães RES, et al. Pulmonary hypertension evaluation by Doppler echocardiogram in children and adolescents with mouth breathing syndrome. Braz J Otorhinolaryngol. 2017 MayJun;83(3):292-8.

8 Şimşek E, Şimşek Z, Taş MH, Kucur C, Günay $\mathrm{E}, \ddot{U} c ̧ u ̈ n c u ̈ H$. Evaluation of right ventricular functions in patients with nasal polyposis: an observational study. Anatol J Cardiol. 2013; 13:251-6.

9 Bayrak P, Kirmaz C, Sekuri C, Yuksel H. Is pulmonary arterial pressure affected by allergic rhinitis with nasal obstruction? Asian Pac J Allergy Immunol. 2007 JunSep;25(2-3): 121-6.

10 Yüksel H, Coşkun Ş, Onağ A. Doppler echocardiographic evaluation of pulmonary arterial pressure in children with allergic rhinitis. Int J Pediatr Otorhinolaryngol. 2001;60(1): 21-7.

11 Reisli I, Oran B, Baspinar O, Baysal T, Karaaslan S. Pulmonary arterial pressure in children with allergic rhinitis. Am J Rhinol. 2004 JulAug;18(4):227-32.
12 Ferreira Nader CMF, Capanema FD, Franco LP, Meira ZMA, Galvão CP, Ramos VM, et al. Pulmonary arterial pressure and nasal obstruction in mouth-breathing children: similarities between adenotonsillar hypertrophy and allergic rhinitis. Int Forum Allergy Rhinol. 2021;11(2):128-35.

13 Lima MS, Nader CMFF, Franco LP, Meira ZMA, Capanema FD, Guimarães RES, et al. Pulmonary hypertension evaluation by Doppler echocardiogram in children and adolescents with mouth breathing syndrome. Braz J Otorhinolaryngol. 2017 MayJun;83(3):292-8.

14 Aukema AA, Mulder PG, Fokkens WJ. Treatment of nasal polyposis and chronic rhinosinusitis with fluticasone propionate nasal drops reduces need for sinus surgery. J Allergy Clin Immunol. 2005;115(5):1017-23.

15 Stjärne P, Mösges R, Jorissen M, Passàli D, Bellussi L, Staudinger $\mathrm{H}$, et al. A randomized controlled trial of mometasone furoate nasal spray for the treatment of nasal polyposis. Arch Otolaryngol Head Neck Surg. 2006; 132(2):179-85. 